\title{
Multiconsensus of Second-Order Multiagent Networks via Pulse-Modulated Intermittent Control
}

\author{
Ming Chi, Xu-Long Wang $(\mathbb{D}$, Ding-Xin He $\mathbb{D}$, and Zhi-Wei Liu \\ Key Laboratory of Image Processing and Intelligent Control, School of Artificial Intelligence and Automation, \\ Huazhong University of Science and Technology, Wuhan 430074, China
}

Correspondence should be addressed to Zhi-Wei Liu; zwliu@hust.edu.cn

Received 27 August 2019; Accepted 21 October 2019; Published 7 April 2020

Academic Editor: Qingling Wang

Copyright (c) 2020 Ming Chi et al. This is an open access article distributed under the Creative Commons Attribution License, which permits unrestricted use, distribution, and reproduction in any medium, provided the original work is properly cited.

This paper studies the multiconsensus problem of multiagent networks based on sampled data information via the pulsemodulated intermittent control (PMIC) which is a general control framework unifying impulsive control, intermittent control, and sampling control. Two kinds of multiconsensus, including stationary multiconsensus and dynamic multiconsensus of multiagent networks, are taken into consideration in such control framework. Based on the eigenvalue analysis and algebraic graph theory, some necessary and sufficient conditions on the feedback gains and the control period are established to ensure the multiconsensus. Finally, several simulation results are included to show the theoretical results.

\section{Introduction}

With the rapid development of complex network theory, scholars use complex networks to describe multiagent systems. If each agent is taken as a node in a multiagent system and the communication or cooperative relationship between agents is regarded as an edge, then the multiagent network can be simplified into a network. We call such a network with intelligent nodes a multiagent network. In daily life, the multiagent network is also ubiquitous, such as smart grid [1], economic dispatch [2], and social network [3]. Multiagent network is one of the most widely used types in complex networks, which generates complex swarm behaviors such as consensus [4], tracking [5], and flocking [6] through the interactions between agents or between agents and the environment. Scholars have developed a strong interest in the clustering behavior of multiagent networks and have obtained some meaningful results, which have been applied to traffic control $[7,8]$, flexible manufacturing $[9,10]$, collaborative expert system [11], intelligent robots, and other fields $[12,13]$.

A systematical framework of consensus problems was established for multiagent networks with the first-order integrator dynamics in [14]. In reality, a number of agents require a double-integrator dynamics. The progress of algorithms from the first-order integrator to the double-integrator dynamics is nontrivial [15-17]. It is worth mentioning that connectivity of communication topologies cannot guarantee consensus of multiagent networks with double-integrator dynamics. In many practical systems, when the multiagent cooperates to complete a complex task, the evolution of the multiagent system shows multiconsensus or multitracking behaviors in some stages due to different task assignments or changes in the environment of the multiagent. Yu and Wang [18-20] proposed the concept of multiconsensus in multiagent systems. Han et al. [21, 22] systematically studied the problem of multiconsensus of second-order multiagent systems. Guan et al. [22] studied the multiconsensus problem of second-order multiagent systems based on sampled position data.

A variety of control methods have been proposed to drive multiagent systems into a consensus, including sampling control [23, 24], impulsive control [25-27], and adaptive control [28-30]. Among them, the sampling control has also attracted much attention from many scholars. The sampling control has been widely used and 
achieved good results [23, 24, 31]. Liu et al. presented a method called the pulse-modulated intermittent control [32], which overcomes the drawback of the sampling control and the pulse control. Such kinds of control can select the appropriate control period and the pulse function according to the specific situation of the system.

Most consensus algorithms consider impulse control or continuous state feedback. When continuous state feedback is used in the algorithm, we need to continuously sample the states of agents over a period of time. However, in most practical systems, the sampling system is not suitable or even able to work continuously in a continuous sampling period. For example, in a driverless system, it is difficult for each vehicle to maintain continuous control output, and the sensors on the vehicle do not work all the time, allowing for fuel economy and other reasons. Impulse control will make the state variable of the controlled object reach a certain value instantaneously, which is determined by impulse control protocol. Considering the control mechanism of the impulse control, the states of agents in some systems cannot be changed instantaneously, such as the rotation speed of four-rotor UAV. Therefore, we need to find a more practical and effective control strategy. Liu et al. [32] proposed the pulse-modulated intermittent control, which can well solve such problems. So, we used this control scheme to solve multiconsensus problems.

We mainly study the multiconsensus problem of multiagent systems based on the pulse-modulated intermittent control in this paper. Based on the basic knowledge of theory of algebraic graph and matrix, we give the sufficient and necessary conditions for second-order multiagent systems to achieve stationary multiconsensus and dynamic multiconsensus under the control method of the pulse-modulated intermittent control. The following sections of the paper are organized as follows. Section 2 gives some basic theorems and some mathematical symbols and there is a brief introduction of the pulse-modulated intermittent control. Section 3 gives the sufficient and necessary conditions for the second-order multiagent systems to reach multiconsensus under PMIC. Section 4 shows two simulation results. The main conclusions of this paper are summarized in Section 5.

\section{Preliminaries and Problem Formulation}

2.1. Preliminaries. $G=(V, E, A)$ is a weighted digraph, where $V=\{1, \ldots, N\}$ is a vertex set, $E \subseteq V \times V$ is a link set, and $A=\left[a_{i j}\right] \in \mathbb{R}^{n \times n}$ is a nonnegative weighted adjacency matrix. The information flow from vertex $j$ to vertex $i$ is represented by a directed link. The elements of matrix $A$ are described as follows: $a_{i j}>0$ if $e_{i j} \in E$ and $a_{i j}=0$, otherwise. Furthermore, it is considered to be $a_{i i}=0$ for all $i \in V$.

Definition 1. A network $G^{l}=\left(V^{l}, E^{l}, A^{l}\right)$ is a subnetwork of $G=(V, E, A)$, where $V^{l} \subseteq V, E^{l} \subseteq E$, and $A^{l}$ is a adjacency matrix of $G^{l}$.

A network $G=(V, E, A)$ consists of $m$ subnetworks $G^{l}=$ $\left(V^{l}, E^{l}, A^{l}\right)$ with $n_{l}(l=1, \ldots, N)$ vertices. It is assumed that a certain node can only belong to a subnetwork, that is, the node sets of different subnetworks have no intersection.
The block form of the weighted adjacency matrix $A$ is established by

$$
A=\left[\begin{array}{cccc}
A_{11} & A_{12} & \cdots & A_{1 m} \\
A_{21} & A_{22} & \cdots & A_{2 m} \\
\vdots & \vdots & \ddots & \vdots \\
A_{m 1} & A_{m 2} & \cdots & A_{m m}
\end{array}\right] .
$$

Based on the adjacency matrix $A$, we need to design a matrix $\bar{L} \in \mathbb{R}^{N \times N}$.

Let $A=\left[a_{i j}\right] \in \mathbb{R}^{p \times q}, \quad A \geq 0, \quad B=\left[b_{i j}\right] \in \mathbb{R}^{p \times q}, \quad$ and $C=\left[c_{i}\right] \in \mathbb{R}^{p}$.

(1) $\Theta: A \longmapsto B$, where $b_{i j} \in\left\{-a_{i j}, 0, a_{i j}\right\}$

(2) $F: B \longmapsto c$, where $c_{i}=\sum_{j=1}^{q} b_{i j}$

$$
\bar{L}=\left[\begin{array}{cccc}
L_{11} & \bar{L}_{12} & \cdots & \bar{L}_{1 m} \\
\bar{L}_{21} & L_{22} & \cdots & \bar{L}_{2 m} \\
\vdots & \vdots & \ddots & \vdots \\
\bar{L}_{m 1} & \bar{L}_{m 2} & \cdots & L_{m m}
\end{array}\right] \text {, }
$$

where $L_{k k}$ is the Laplacian matrix of $A_{k k}$. For any $i \neq j, \bar{L}_{i j}=$ $\Theta\left(A_{i j}\right)$ with $F\left(\bar{L}_{i j}\right)=0_{n_{i}}$ is true. $\bar{L}$ has $m$ eigenvalues of 0 , and $m$ eigenvectors $p_{1}=\left[1_{n_{1}}^{T}, 0_{N-n_{1}}^{T}\right]^{T}, \quad p_{2}=\left[0_{n_{1}}^{T}, 1_{n_{2}}^{T}\right.$, $\left.0_{N-n_{1}-n_{2}}^{T}\right]^{T}, \ldots$, and $p_{m}=\left[0_{N-n_{m}}^{T}, 1_{n_{m}}^{T}\right]^{T}$ related to the 0 eigenvalue. In addition to, $\lambda_{i}$ is defined as the $i$ th eigenvalue of $\bar{L}$.

Remark 1. The mapping $\Theta: A \longmapsto B$ is used when one designs $\bar{L}$. The value of $b_{i j}$ follows the following principles in the selection process (priority from top to bottom):

(1) $F\left(\bar{L}_{i j}\right)=0_{n_{i}}$ for $i \neq j$

(2) The case of $b_{i j}=0$ should be as few as possible

The control input of agent $i$ is denoted by $u_{i} \in \mathscr{R}^{f}$, respectively, position by $p_{i} \in \mathscr{R}^{f}$, and velocity by $v_{i} \in \mathscr{R}^{f}$. Consider a multiagent system with second-order dynamics as

$$
\begin{aligned}
\dot{p}_{i}(t) & =v_{i}(t), \\
\dot{v}_{i}(t) & =u_{i}(t) .
\end{aligned}
$$

Definition 2 (see [21]). For second-order integral multiagent systems, consensus can be classified into two categories, which are stationary consensus and dynamic consensus.

(1) The agents reach a stationary consensus in their respective subnetworks for any initial values if the system satisfies

$$
\left\{\begin{array}{l}
\lim _{t \longrightarrow \infty}\left\|p_{i}(t)-p_{j}(t)\right\|=0, \quad \forall i, j \in V^{l}, \\
\lim _{t \longrightarrow \infty} v_{i}(t)=0,
\end{array}\right.
$$

where $l$ is the label of subnetworks. 
(2) The agents reach a dynamic consensus in their respective subnetworks for any initial values if the system satisfies

$$
\left\{\begin{array}{l}
\lim _{t \longrightarrow \infty}\left\|p_{i}(t)-p_{j}(t)\right\|=0 \\
\lim _{t \rightarrow \infty}\left\|v_{i}(t)-v_{j}(t)\right\|=0, \quad \forall i, j \in V^{l} \\
0<\sup _{t \longrightarrow \infty}\left\|v_{i}(t)\right\| \leq \varphi_{l}
\end{array}\right.
$$

where $l$ is the label of the subnetwork and $\varphi_{l}$ is a positive constant.

Lemma 1 (see [21]). Multiagent system achieves a stationary multiconsensus if each subsystem reaches a stationary consensus. Multiagent system achieves a dynamic multiconsensus if each subsystem reaches the dynamic consensus.

Lemma 2. Complex characteristic polynomial $R(z)=z^{2}+$ $r z+s$ is Hurwitz stable if and only if $\operatorname{Re}(r)>0$ and $\operatorname{Re}(r) \operatorname{Im}(r) \operatorname{Im}(s)+\operatorname{Re}^{2}(r) \operatorname{Re}(s)-\operatorname{Im}^{2}(s)>0$.

2.2. Pulse-Modulated Intermittent Control. For working out the multiconsensus issue of the multiagent system of (3), the control protocol is propounded in [22] as

$$
u(t)=-\alpha P p(t)-\beta Q v(t) .
$$

Remark 2. Let $P=\bar{L}$ and $Q=I_{N}$ when the system converges to the stationary multiconsensus. Let $P=\bar{L}$ and $Q=\bar{L}$ when the system converges to the dynamic multiconsensus.

The following control protocol is proposed for solving the multiconsensus issue of (3) via the pulse-modulated intermittent control:

$$
u(t)=-a\left(t-t_{k}\right)\left(\alpha P p\left(t_{k}\right)+\beta Q v\left(t_{k}\right)\right),
$$

where $u(t)=\left(u_{1}(t), u_{2}(t), \ldots, u_{N}(t)\right)^{T}, p\left(t_{k}\right)=\left(p_{1}\left(t_{k}\right)\right.$, $\left.p_{2}\left(t_{k}\right), \ldots, p_{N}\left(t_{k}\right)\right)^{T}, \quad v\left(t_{k}\right)=\left(v_{1}\left(t_{k}\right), v_{2}\left(t_{k}\right), \ldots, \quad v_{N}\right.$ $\left.\left(t_{k}\right)\right)^{T}, t_{k}<t \leq t_{k+1}$, and $\alpha$ and $\beta$ are nonnegative feedback gains, and let $h=t_{k+1}-t_{k}$. The pulse function which depends on the actual control system is described as

$$
a(t)= \begin{cases}\hat{a}(t), & t \in(0, d], \\ 0, & t \notin(0, d],\end{cases}
$$

where $\widehat{a}(t)$ is a scale function which is continuous and piecewise, and $d<h$ is the duration time that the pulse function takes effect. $\left(t_{k}+d, t_{k+1}\right]$ is the rest interval and $\left(t_{k}, t_{k}+d\right]$ is the control interval [32].

According to (3) and (7), one can obtain that

$$
\begin{cases}{\left[\begin{array}{c}
\dot{p}(t) \\
\dot{v}(t)
\end{array}\right]=\left[\begin{array}{cc}
0_{N} & I_{N} \\
0_{N} & 0_{N}
\end{array}\right]\left[\begin{array}{c}
p(t) \\
v(t)
\end{array}\right]+\widehat{a}\left(t-t_{k}\right)\left[\begin{array}{cc}
0_{N} & 0_{N} \\
-\alpha P & -\beta Q
\end{array}\right]\left[\begin{array}{c}
p\left(t_{k}\right) \\
v\left(t_{k}\right)
\end{array}\right],} & t \in\left(t_{k}, t_{k}+d\right], \\
{\left[\begin{array}{c}
\dot{p}(t) \\
\dot{v}(t)
\end{array}\right]=\left[\begin{array}{ll}
0_{N} & I_{N} \\
0_{N} & 0_{N}
\end{array}\right]\left[\begin{array}{c}
p(t) \\
v(t)
\end{array}\right],} & t \notin\left(t_{k}, t_{k}+d\right] .\end{cases}
$$

Let

$$
\begin{aligned}
x(t) & \triangleq\left[\begin{array}{l}
p(t) \\
v(t)
\end{array}\right], \\
A & \triangleq\left[\begin{array}{ll}
0_{N} & I_{N} \\
0_{N} & 0_{N}
\end{array}\right], \\
B & \triangleq\left[\begin{array}{cc}
0_{N} & 0_{N} \\
-\alpha P & -\beta Q
\end{array}\right] .
\end{aligned}
$$

Then, the state space equation of the second-order system is described as

$$
\begin{cases}\dot{x}(t)=A x(t)+\hat{a}\left(t-t_{k}\right) B x\left(t_{k}\right), & t \in\left(t_{k}, t_{k}+d\right), \\ \dot{x}(t)=A x(t), & t \notin\left(t_{k}, t_{k}+d\right) .\end{cases}
$$

The solution of (11) can be expressed as

$$
x(t)=e^{A\left(t-t_{0}\right)} x\left(t_{0}\right)+e^{A t} \int_{t_{0}}^{t} e^{-A \tau} \widehat{a}\left(\tau-t_{k}\right) B x\left(t_{k}\right) \mathrm{d} \tau .
$$

Let

$$
\begin{aligned}
t & =t_{k}+d, \\
\tau & =s \\
d_{1} & =\int_{0}^{d} \widehat{a}(s) \mathrm{d} s, \\
d_{2} & =\int_{0}^{d} s \widehat{a}(s) \mathrm{d} s .
\end{aligned}
$$

By simple calculations, the above equation can be expressed as

$x\left(t_{k}+d\right)=e^{A d} x\left(t_{k}\right)+e^{A\left(t_{k}+d\right)} \int_{t_{k}}^{t_{k}+d} e^{-A s} \widehat{a}\left(s-t_{k}\right) B x\left(t_{k}\right) \mathrm{d} s$.

Noticing that $A^{2}=0$, one can obtain that

$$
x\left(t_{k}+d\right)=\left(I_{2 N}+d A+d_{1} B+\left(d d_{1}-d_{2}\right) A B\right) x\left(t_{k}\right) .
$$

Notice that $x\left(t_{k+1}\right)=x\left(t_{k}+h\right)$. Then, the system can be discretely expressed as

$$
x\left(t_{k+1}\right)=\left(I_{2 N}+h A+d_{1} B+\left(h d_{1}-d_{2}\right) A B\right) x\left(t_{k}\right) .
$$

Let 


$$
\begin{aligned}
\Gamma & \triangleq\left(I_{2 N}+h A+d_{1} B+\left(h d_{1}-d_{2}\right) A B\right) \\
& =\left[\begin{array}{cc}
I_{N}-\left(h d_{1}-d_{2}\right) \alpha P & h I_{N}-\left(h d_{1}-d_{2}\right) \beta Q \\
-\alpha d_{1} P & I_{N}-\beta d_{1} Q
\end{array}\right] .
\end{aligned}
$$

\section{Convergence Analysis of the Controlled Multiagent Networks}

In this section, there are two problems to be illuminated before the proof. One is that, as mentioned in Remark 2, in this section, we will choose different $P$ and $Q$ according to the type of multiconsensus. Another is that the proof of dynamic multiconsensus of the multiagent network is basically similar to the proof of stationary multiconsensus of the multiagent network, so it is omitted here.

3.1. Stationary Multiconsensus. For working out the stationary multiconsensus problem, we choose matrices $P=\bar{L}$ and $Q=I_{N}$ in (6), and one can obtain the matrix $\Gamma_{1}$ :

$$
\Gamma_{1}=\left[\begin{array}{cc}
I_{N}-\left(h d_{1}-d_{2}\right) \alpha \bar{L} & h I_{N}-\left(h d_{1}-d_{2}\right) \beta I_{N} \\
-\alpha d_{1} \bar{L} & I_{N}-\beta d_{1} I_{N}
\end{array}\right] .
$$

When studying the problem, the eigenvalue of $\Gamma_{1}$ is found to play a significant role in the realization of multiconsensus of (6). It is also found that there is a certain correlation between the eigenvalues of $\Gamma_{1}$ and $\bar{L}$. Due to the complexity of $\Gamma_{1}$ corresponding to the directed topology, in the following study, we avoided starting with $\Gamma_{1}$ directly but first studied the analytic relationship between $\Gamma_{1}$ and $\bar{L}$.

Theorem 1. The eigenvalues of $\Gamma_{1}$ are encircled by unit circle or equivalent to 1 , and the algebraic multiplicity of 1 is $m$ if and only if $\lambda_{1}=\lambda_{2}=\cdots=\lambda_{m}=0$, and

$$
\begin{aligned}
& \frac{\beta}{\alpha}>\max _{\lambda_{i} \neq 0}\left(\frac{d_{2}\left|\lambda_{i}\right|^{2}}{d_{1} \operatorname{Re}\left(\lambda_{i}\right)}\right), \\
& \mathrm{d}<h<\min _{\lambda_{i} \neq 0} \frac{M^{2} N-M C}{M^{2} D+E D}, \\
& 0<d_{1}<\frac{2}{\beta},
\end{aligned}
$$

where

$$
\begin{aligned}
M & =2 \beta d_{1} \operatorname{Re}\left(\lambda_{i}\right)-2 \alpha d_{2}\left|\lambda_{i}\right|^{2}, \\
N & =2 \alpha d_{2}\left|\lambda_{i}\right|^{2}+\left(4-2 \beta d_{1}\right) \operatorname{Re}\left(\lambda_{i}\right), \\
C & =2 \beta d_{1}\left(\operatorname{Im}\left(\lambda_{i}\right)\right)^{2}\left(2 \beta d_{1}-4\right), \\
D & =\alpha d_{1}\left|\lambda_{i}\right|^{2}, \\
E & =\left(\left(2 \beta d_{1}-4\right) \operatorname{Im}\left(\lambda_{i}\right)\right)^{2} .
\end{aligned}
$$

Proof. Given the characteristic polynomial of $\Gamma_{1}$ by

$$
\begin{aligned}
\operatorname{det}\left(s I_{2 N}-\Gamma_{1}\right)= & \operatorname{det}\left((s-1)\left(s-1+\beta d_{1}\right) I_{N}+\alpha d_{1} h \bar{L}\right. \\
& \left.+\alpha\left(h d_{1}-d_{2}\right)(s-1) \bar{L}\right) \\
= & \prod_{i=1}^{N}\left(s^{2}+\left(\beta d_{1}+\alpha \lambda_{i} h d_{1}-\alpha \lambda_{i} d_{2}-2\right) s\right. \\
& \left.+\alpha \lambda_{i} d_{2}-\beta d_{1}+1\right) .
\end{aligned}
$$

The solutions of characteristic equation $\operatorname{det}\left(s I_{2 N}-\Gamma_{1}\right)=$ 0 satisfy

$$
s^{2}+\left(\beta d_{1}+\alpha \lambda_{i} h d_{1}-\alpha \lambda_{i} d_{2}-2\right) s+\alpha \lambda_{i} d_{2}-\beta d_{1}+1=0 .
$$

The above formula shows that $\Gamma_{1}$ has two eigenvalues corresponding to each $\lambda_{i}$. Consider that $s_{1}=1$ and $s_{2}=1-$ $\beta d_{1}$ if and only if $\lambda_{i}=0$, which indicates that $\left|s_{2}\right|<1$ if and only if

$$
0<d_{1}<\frac{2}{\beta} .
$$

Then, it is focused on the conditions for $\lambda_{i} \neq 0$ that the eigenvalues of $\Gamma_{1}$ are encircled by the unit circle. Applying a bilinear transformation, $z=s+1 / s-1$, an updated polynomial is found as

$$
R(z)=z^{2}+\frac{2\left(\beta d_{1}-\alpha \lambda_{i} d_{2}\right)}{\alpha \lambda_{i} h d_{1}} z+\frac{2 \alpha \lambda_{i} d_{2}-2 \beta d_{1}-4}{\alpha \lambda_{i} h d_{1}}-1 .
$$

Considering the bilinear transformation, it follows that, for $\lambda_{i} \neq 0$, the eigenvalues of $\Gamma_{1}$ are encircled by the unit circle if and only if $R(z)$ (26) is Hurwitz stable.

Comparing the terms in polynomial (26) with the terms in Lemma 2, it is obtained that

$$
\begin{aligned}
& \operatorname{Re}(r)=\frac{2 \beta d_{1} \operatorname{Re}\left(\lambda_{i}\right)-2 \alpha d_{2}\left|\lambda_{i}\right|^{2}}{\alpha\left|\lambda_{i}\right|^{2} h d_{1}}, \\
& \operatorname{Im}(r)=\frac{-2 \beta d_{1} \operatorname{Im}\left(\lambda_{i}\right)}{\alpha\left|\lambda_{i}\right|^{2} h d_{1}}, \\
& \operatorname{Re}(s)=\frac{2 \alpha d_{2}\left|\lambda_{i}\right|^{2}+\left(4-2 \beta d_{1}\right) \operatorname{Re}\left(\lambda_{i}\right)-\alpha\left|\lambda_{i}\right|^{2} h d_{1}}{\alpha\left|\lambda_{i}\right|^{2} h d_{1}}, \\
& \operatorname{Im}(s)=\frac{-\left(4-2 \beta d_{1}\right) \operatorname{Im}\left(\lambda_{i}\right)}{\alpha\left|\lambda_{i}\right|^{2} h d_{1}} .
\end{aligned}
$$

Applying Lemma 2 and considering $d<h$, then $R(z)$ is Hurwitz stable if and only if

$$
\begin{aligned}
& \frac{\beta}{\alpha}>\max _{\lambda_{i} \neq 0}\left(\frac{d_{2}\left|\lambda_{i}\right|^{2}}{d_{1} \operatorname{Re}\left(\lambda_{i}\right)}\right), \\
& \mathrm{d}<h<\min _{\lambda_{i} \neq 0} \frac{M^{2} N-M C}{M^{2} D+E D},
\end{aligned}
$$

where 


$$
\begin{aligned}
& M=2 \beta d_{1} \operatorname{Re}\left(\lambda_{i}\right)-2 \alpha d_{2}\left|\lambda_{i}\right|^{2}, \\
& N=2 \alpha d_{2}\left|\lambda_{i}\right|^{2}+\left(4-2 \beta d_{1}\right) \operatorname{Re}\left(\lambda_{i}\right), \\
& C=2 \beta d_{1}\left(\operatorname{Im}\left(\lambda_{i}\right)\right)^{2}\left(2 \beta d_{1}-4\right), \\
& D=\alpha d_{1}\left|\lambda_{i}\right|^{2}, \\
& E=\left(\left(2 \beta d_{1}-4\right) \operatorname{Im}\left(\lambda_{i}\right)\right)^{2} .
\end{aligned}
$$

The proof is completed.

Theorem 2. System described by (3) asymptotically reaches stationary multiconsensus of the multiagent network under PMIC protocol (7) among which $P=\bar{L}$ and $Q=I_{n}$ if and only if inequalities (19), (20), and (21) are satisfied and $\lambda_{1}=\lambda_{2}=\cdots=\lambda_{m}=0$.

Proof. Taking Theorem 1 into consideration, it is indicated that system (3) asymptotically reaches the stationary multiconsensus network if and only if the eigenvalues of $\Gamma_{1}$ are encircled by unit circle or equivalent to 1 and the algebraic multiplicity of 1 is $m$.

3.1.1. Sufficiency. $\Gamma_{1}$ has $m$ eigenvalues 1 , with linearly independent eigenvectors expressed as $\phi_{1}=[1,0]^{T} \otimes p_{1}$, $\phi_{2}=[1,0]^{T} \otimes p_{2}, \ldots l, \phi_{m}=[1,0]^{T} \otimes p_{m} . \Gamma_{1}$ can be described in the following form as

$$
\Gamma_{1}=\Phi_{1}\left[\begin{array}{ll}
I_{m} & \\
& J
\end{array}\right] \Phi_{1}^{-1}
$$

where $\Phi_{1}$ is a nonsingular matrix and $\Phi_{1}=\left[\phi_{1}, \ldots, \phi_{2 n}\right]$. The eigenvalues of $\Gamma_{1}$ within the unit circle correspond to the Jordan block matrix $J$. As $t$ goes to infinity,

$$
\lim _{l \longrightarrow \infty} \Gamma_{1}^{l}=\Phi_{1}\left[\begin{array}{ll}
I_{m} & \\
& O_{2 n-m}
\end{array}\right] \Phi_{1}^{-1} .
$$

Let $\Phi_{1}^{-1}=\left[\varphi_{1}^{T}, \ldots, \varphi_{2 n}^{T}\right]^{T}$, where $\varphi_{i} \in \mathbb{C}^{1 \times 2 n}$ and $\varphi_{i} \phi_{i}=1(i=1, \ldots, 2 n)$. Then, use the Kronecker product to write the above equation as follows:

$$
\prod_{1} \triangleq \lim _{l \longrightarrow \infty} \Gamma_{1}^{l}=\left[\begin{array}{c}
1_{n_{1}} \otimes \varphi_{1} \\
\vdots \\
1_{n_{m}} \otimes \varphi_{m} \\
O_{n \times 2 n}
\end{array}\right]
$$

The steady states of system (3) can be written as

$$
\left[\begin{array}{l}
p(t) \\
v(t)
\end{array}\right]=\prod_{1}\left[\begin{array}{l}
p\left(t_{0}\right) \\
v\left(t_{0}\right)
\end{array}\right] .
$$

Denote the index of the subnetwork in which agent $i$ lies by $\hat{i}$. As $t \longrightarrow \infty$, one can obtain

$$
\begin{aligned}
& p_{i}(t) \longrightarrow \varphi \widehat{i}\left[\begin{array}{l}
p\left(t_{0}\right) \\
v\left(t_{0}\right)
\end{array}\right], \\
& v_{i}(t) \longrightarrow 0 .
\end{aligned}
$$

Then, it is easily obtained that

$$
\left\{\begin{array}{l}
\lim _{t \rightarrow \infty}\left\|p_{i}(t)-p_{j}(t)\right\|=0, \quad \forall i, j \in V^{l}, \\
\lim _{t \rightarrow \infty} v_{i}(t)=0 .
\end{array}\right.
$$

The above shows that each agent within the same subnetwork reaches consensus. According to Lemma 1, the stationary multiconsensus issue of the multiagent networks is considered to have worked out with PMIC protocol (7).

3.1.2. Necessity. If system (3) achieves stationary multiconsensus, it follows that $\lim _{l \longrightarrow \infty} \Gamma_{1}$ is a matrix with rank $m$, which indicates that the eigenvalues of $\Gamma_{1}$ are encircled by unit circle or equivalent to 1 , and the algebraic multiplicity of 1 is $m$.

3.2. Dynamic Multiconsensus. For working out the dynamic multiconsensus problem, we choose matrices $P=\bar{L}$ and $Q=$ $\bar{L}$ in (6), and one can obtain the matrix $\Gamma_{2}$ :

$$
\Gamma_{2}=\left[\begin{array}{cc}
I_{N}-\left(h d_{1}-d_{2}\right) \alpha \bar{L} & h I_{N}-\left(h d_{1}-d_{2}\right) \beta \bar{L} \\
-\alpha d_{1} \bar{L} & I_{N}-\beta d_{1} \bar{L}
\end{array}\right] .
$$

Theorem 3. The eigenvalues of $\Gamma_{2}$ are encircled by unit circle or equivalent to 1 , and the algebraic multiplicity of 1 is $2 m$ if and only if $\lambda_{1}=\lambda_{2}=\cdots=\lambda_{m}=0$, and

$$
\begin{aligned}
& \frac{\beta}{\alpha}>\frac{d_{2}}{d_{1}}, \\
& d<h<\min _{\lambda_{i} \neq 0} \frac{M_{1}^{3}\left|\lambda_{i}\right|^{4}+4 \operatorname{Re}\left(\lambda_{i}\right)\left|\lambda_{i}\right|^{2} M_{1}^{2}}{16 \alpha d_{1} \operatorname{Im}^{2}\left(\lambda_{i}\right)+\alpha d_{1} M_{1}^{2}\left|\lambda_{i}\right|^{4}}
\end{aligned}
$$

where $M_{1}=2 \alpha d_{2}-2 \beta d_{1}$.

Theorem 4. System described by (3) asymptotically reaches dynamic multiconsensus of the multiagent network under PMIC protocol (7) with $P=\bar{L}$ and $Q=\bar{L}$ if and only if $\lambda_{1}=\lambda_{2}=\cdots=\lambda_{m}=0$, and (37) and (38) hold.

\section{Simulation}

Here are the two types of simulations to illustrate the validity of the results presented in the previous section. Matrix $A$ of directed topology is designed as 


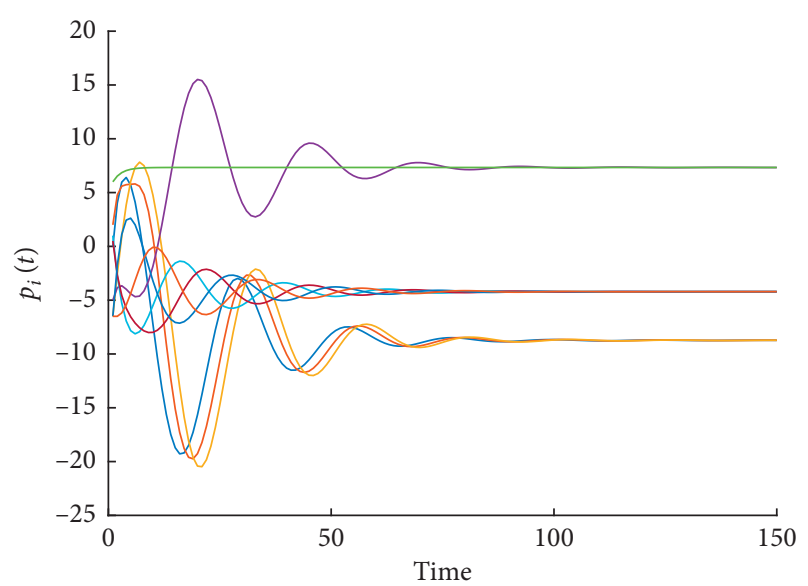

FIGURE 1: Stationary multiconsensus: positions of agents.

$$
A=\left[\begin{array}{lllllllll}
0 & 0 & 0 & 1 & 1 & 1 & 0 & 0 & 1 \\
3 & 0 & 1 & 0 & 0 & 0 & 0 & 0 & 0 \\
1 & 1 & 0 & 0 & 0 & 0 & 0 & 0 & 0 \\
1 & 0 & 1 & 0 & 0 & 0 & 0 & 0 & 0 \\
1 & 0 & 0 & 0 & 0 & 0 & 0 & 0 & 0 \\
0 & 0 & 0 & 0 & 0 & 0 & 0 & 0 & 1 \\
0 & 0 & 0 & 0 & 0 & 1 & 0 & 0 & 0 \\
0 & 0 & 0 & 0 & 0 & 0 & 1 & 0 & 0 \\
0 & 0 & 0 & 0 & 0 & 0 & 0 & 1 & 0
\end{array}\right]
$$

This network obviously has three subnetworks: (1) $V_{1}=\{1,2,3\} ;(2) V_{2}=\{4,5\}$; and (3) $V_{3}=\{6,7,8,9\}$. The matrix $\bar{L}$ is designed by

$$
\bar{L}=\left[\begin{array}{ccccccccc}
0 & 0 & 0 & -1 & 1 & -1 & 0 & 0 & 1 \\
-3 & 4 & -1 & 0 & 0 & 0 & 0 & 0 & 0 \\
-1 & -1 & 2 & 0 & 0 & 0 & 0 & 0 & 0 \\
1 & 0 & -1 & 0 & 0 & 0 & 0 & 0 & 0 \\
0 & 0 & 0 & 0 & 0 & 0 & 0 & 0 & 0 \\
0 & 0 & 0 & 0 & 0 & 1 & 0 & 0 & -1 \\
0 & 0 & 0 & 0 & 0 & -1 & 1 & 0 & 0 \\
0 & 0 & 0 & 0 & 0 & 0 & -1 & 1 & 0 \\
0 & 0 & 0 & 0 & 0 & 0 & 0 & -1 & 1
\end{array}\right] .
$$

The eigenvalues of $\bar{L}$ are $\lambda_{1}=4.4567, \lambda_{2}=2, \lambda_{3}=1+\mathbf{i}$, $\lambda_{4}=1-\mathbf{i}, \lambda_{5}=0.7717+0.7256 \mathbf{i}, \lambda_{6}=0.7717-0.7256 \mathbf{i}$, and $\lambda_{7}=\lambda_{8}=\lambda_{9}=0$.

We can choose the function $\hat{a}(t)=(t / d)$, and we can choose the control period $d=0.2$, and then $d_{1}=0.1$ and $d_{2}=0.01333$.

For stationary multiconsensus, it follows from (19) and (21) that $(\beta / \alpha)>0.5941$ and $\beta<20$. In order to make the simulation look suitable, the feedback gains $\beta=2.5$ and $\alpha=$ 1 are chosen. According to inequality (20), $0.2<h<1.7251$ is obtained, and $h=0.8$ is chosen. The initial state of each

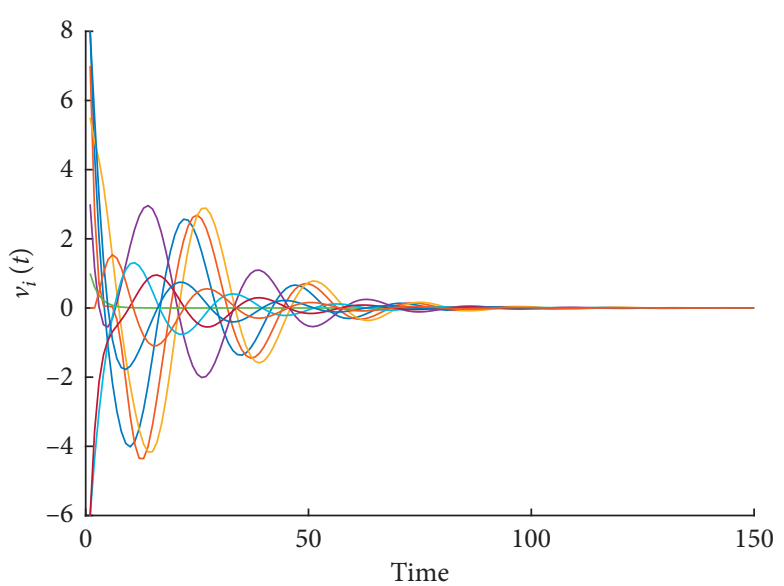

FIgURE 2: Stationary multiconsensus: velocities of agents.

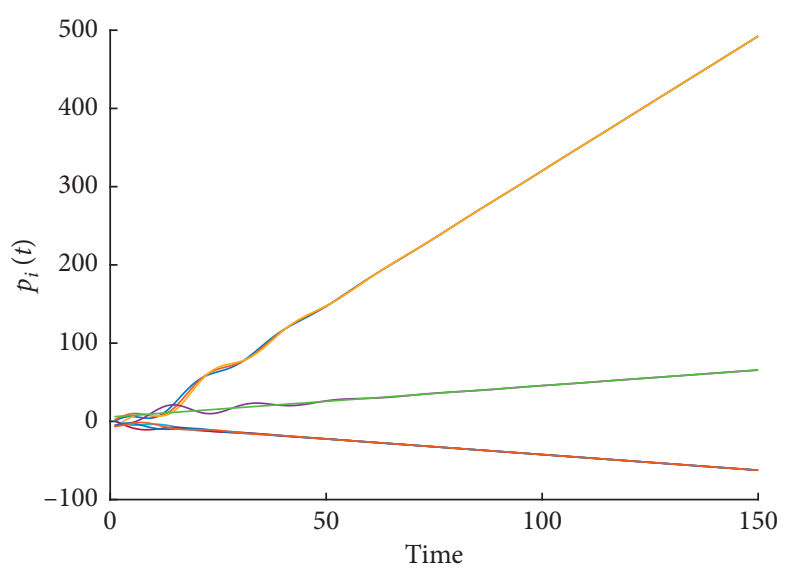

Figure 3: Dynamic multiconsensus: positions of agents.

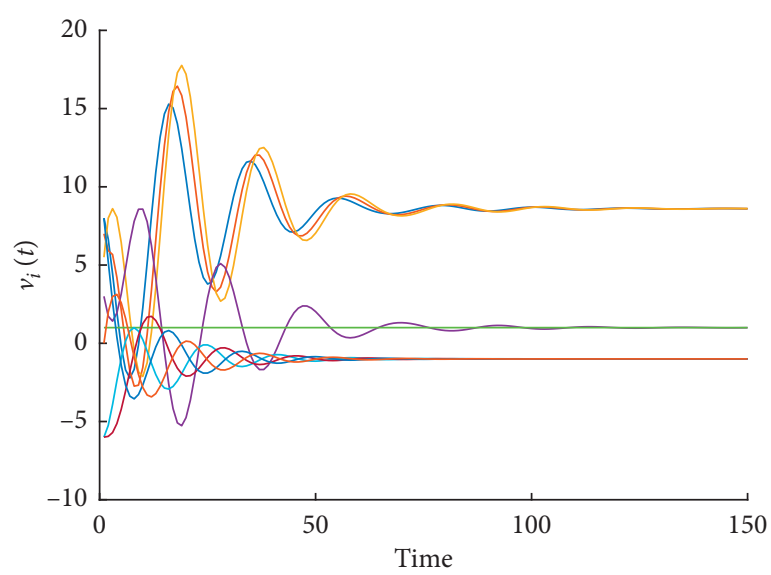

Figure 4: Dynamic multiconsensus: velocities of agents.

agent is randomly selected, and the position and velocity of each agent varying with time are shown in Figures 1 and 2, respectively.

For dynamic multiconsensus, similarly, we can choose the function $\hat{a}(t)=(t / d)$, and we can choose the control 
period $d=0.2$, and then $d_{1}=0.1$ and $d_{2}=0.01333$. According to (10), $(\beta / \alpha)>0.1333$ is obtained, and $\beta=2.5$ and $\alpha=1$ are chosen. According to (38), $0.2<h<0.7380$ is obtained, and $h=0.4$ is chosen. The initial position and velocity of agents are randomly selected, and their values varying with time are shown in Figures 3 and 4, respectively.

\section{Conclusion}

The multiconsensus of second-order multiagent networks with a directed topology is investigated in this paper. A PMIC protocol is proposed to achieve the stationary multiconsensus and the dynamic multiconsensus of the multiagent network. Necessary and sufficient conditions are established to ensure achieving two categories of multiconsensus of the multiagent system. Different from previous studies, a PMIC protocol is applied to multiagent systems, which can adapt our method to more practical systems. In the future research work, we will devote more attention to problems related to second-order time-delay multiagent systems, for example, pulse-modulated intermittent control in multiconsensus with time delay.

\section{Data Availability}

No data were used to support this study.

\section{Conflicts of Interest}

The authors declare that they have no conflicts of interest.

\section{Acknowledgments}

This work was supported partly by the National Natural Science Foundation of China under Grants 61972170 and 61973133.

\section{References}

[1] X. Hu, Z.-W. Liu, G. Wen, X. Yu, and C. Li, "Branch-wise parallel successive algorithm for online voltage regulation in distribution networks," IEEE Transactions on Smart Grid, vol. 10, no. 6, pp. 6678-6689, 2019.

[2] X. Hu, H. Zhou, Z.-W. Liu, X. Yu, and C. Li, "Hierarchical distributed scheme for demand estimation and power reallocation in a future power grid," IEEE Transactions on Industrial Informatics, vol. 13, no. 5, pp. 2279-2290, 2017.

[3] M. Ye, Y. Qin, A. Govaert, B. D. O. Anderson, and M. Cao, "An influence network model to study discrepancies in expressed and private opinions," Automatica, vol. 107, pp. 371-381, 2019.

[4] Z.-W. Liu, G. Wen, X. Yu, Z.-H. Guan, and T. Huang, "Delayed impulsive control for consensus of multiagent systems with switching communication graphs," IEEE Transactions on Cybernetics, pp. 1-11. In press.

[5] G. Wen, T. Huang, W. Yu, Y. Xia, and Z.-W. Liu, "Cooperative tracking of networked agents with a high-dimensional leader: qualitative analysis and performance evaluation," IEEE Transactions on Cybernetics, vol. 48, no. 7, pp. 2060-2073, 2017.

[6] P. Yu, L. Ding, Z.-W. Liu, and Z.-H. Guan, "Leader-follower flocking based on distributed event-triggered hybrid control,"
International Journal of Robust and Nonlinear Control, vol. 26, no. 1, pp. 143-153, 2016.

[7] M. Abdoos, N. Mozayani, and A. L. C. Bazzan, "Holonic multi-agent system for traffic signals control," Engineering Applications of Artificial Intelligence, vol. 26, no. 5-6, pp. 1575-1587, 2013.

[8] P. G. Balaji and D. Srinivasan, "Multi-agent system in urban traffic signal control," IEEE Computational Intelligence Magazine, vol. 5, no. 4, pp. 43-51, 2010.

[9] H. T. N. Nejad, N. Sugimura, K. Iwamura, and Y. Tanimizu, "Multi agent architecture for dynamic incremental process planning in the flexible manufacturing system," Journal of Intelligent Manufacturing, vol. 21, no. 4, pp. 487-499, 2010.

[10] K.-Y. Chen and C.-J. Chen, "Applying multi-agent technique in multi-section flexible manufacturing system," Expert Systems with Applications, vol. 37, no. 11, pp. 7310-7318, 2010.

[11] O. López-Ortega and I. Villar-Medina, "A multi-agent system to construct production orders by employing an expert system and a neural network," Expert Systems with Applications, vol. 36, no. 2, pp. 2937-2946, 2009.

[12] Y. Duan, B. X. Cui, and X. H. Xu, "A multi-agent reinforcement learning approach to robot soccer," Artificial Intelligence Review, vol. 38, no. 3, pp. 193-211, 2012.

[13] C. Chen, W. Gui, L. Wu, Z. Liu, and H. Yan, "Tracking performance limitations of mimo networked control systems with multiple communication constraints," IEEE Transactions on Cybernetics, pp. 1-14. In press.

[14] R. Olfati-Saber and R. M. Murray, "Consensus problems in networks of agents with switching topology and time-delays," IEEE Transactions on Automatic Control, vol. 49, no. 9, pp. 1520-1533, 2004.

[15] G. Xie and L. Wang, "Consensus control for a class of networks of dynamic agents," International Journal of Robust and Nonlinear Control: IFAC-Affiliated Journal, vol. 17, no. 10-11, pp. 941-959, 2007.

[16] H. Hu, L. Yu, G. Chen, and G. Xie, "Second-order consensus of multi-agent systems with unknown but bounded disturbance," International Journal of Control, Automation and Systems, vol. 11, no. 2, pp. 258-267, 2013.

[17] W. Ren and E. Atkins, "Distributed multi-vehicle coordinated control via local information exchange," International Journal of Robust and Nonlinear Control, vol. 17, no. 10-11, pp. 1002-1033, 2007.

[18] J. Yu and L. Wang, "Group consensus of multi-agent systems with undirected communication graphs," in Proceedings of the 2009 7th Asian Control Conference, Hong Kong, China, August 2009.

[19] J. Yu and L. Wang, "Group consensus in multi-agent systems with switching topologies and communication delays," Systems \& Control Letters, vol. 59, no. 6, pp. 340-348, 2010.

[20] J. Yu and L. Wang, "Group consensus of multi-agent systems with directed information exchange," International Journal of Systems Science, vol. 43, no. 2, pp. 334-348, 2012.

[21] G.-S. Han, Z.-H. Guan, X.-M. Cheng, Y. Wu, and F. Liu, "Multiconsensus of second order multiagent systems with directed topologies," International Journal of Control, Automation and Systems, vol. 11, no. 6, pp. 1122-1127, 2013.

[22] Z. H. Guan, G. S. Han, J. Li, D. X. He, and G. Feng, "Impulsive multiconsensus of second-order multiagent networks using sampled position data," IEEE Transactions on Neural Networks \& Learning Systems, vol. 26, no. 11, p. 1, 2015.

[23] W. Yu, L. Zhou, X. Yu, J. Lu, and R. Lu, "Consensus in multiagent systems with second-order dynamics and sampled 
data," IEEE Transactions on Industrial Informatics, vol. 9, no. 4, pp. 2137-2146, 2013.

[24] Y. W. Wang, J. W. Xiao, C. Wen, and Z. H. Guan, "Synchronization of continuous dynamical networks with discrete-time communications," IEEE Transactions on Neural Networks, vol. 22, no. 12, pp. 1979-1986, 2011.

[25] Z.-W. Liu, Z.-H. Guan, X. Shen, and G. Feng, "Consensus of multi-agent networks with aperiodic sampled communication via impulsive algorithms using position-only measurements," IEEE Transactions on Automatic Control, vol. 57, no. 10, pp. 2639-2643, 2012.

[26] Z.-W. Liu, X. Hu, M.-F. Ge, and Y.-W. Wang, "Asynchronous impulsive control for consensus of second-order multi-agent networks," Communications in Nonlinear Science and $\mathrm{Nu}$ merical Simulation, vol. 79, Article ID 104892, 2019.

[27] Z. H. Guan, Z. W. Liu, G. Feng, and Y. W. Wang, "Synchronization of complex dynamical networks with timevarying delays via impulsive distributed control," IEEE Transactions on Circuits \& Systems I Regular Papers, vol. 57, no. 8, pp. 2182-2195, 2010.

[28] Z. Li, G. Wen, Z. Duan, and W. Ren, "Designing fully distributed consensus protocols for linear multi-agent systems with directed graphs," IEEE Transactions on Automatic Control, vol. 60, no. 4, pp. 1152-1157, 2015.

[29] C. L. P. Chen, G. X. Wen, Y. J. Liu, and L. Zhi, "Observerbased adaptive backstepping consensus tracking control for high-order nonlinear semi-strict-feedback multiagent systems," IEEE Transactions on Cybernetics, vol. 46, no. 7, pp. 1591-1601, 2016.

[30] C. L. P. Chen, G. X. Wen, Y. J. Liu, and F. Y. Wang, "Adaptive consensus control for a class of nonlinear multiagent timedelay systems using neural networks," IEEE Transactions on Neural Networks \& Learning Systems, vol. 25, no. 6, pp. 1217-1226, 2014

[31] Z. G. Wu, P. Shi, H. Su, and J. Chu, "Sampled-data synchronization of chaotic lur'e systems with time delays," IEEE Transactions on Neural Networks \& Learning Systems, vol. 24, no. 3, pp. 410-421, 2013.

[32] Z.-W. Liu, X. Yu, Z.-H. Guan, B. Hu, and C. Li, "Pulsemodulated intermittent control in consensus of multiagent systems," IEEE Transactions on Systems, Man, and Cybernetics: Systems, vol. 47, no. 5, pp. 783-793, 2017. 